\title{
Effect of breast-conserving surgery combined with sentinel lymph node biopsy and axillary preservation on the recurrence, metastasis, complications and cosmetic results of early breast cancer patients
}

\author{
Jing Xiang ${ }^{1 *}$, Shiqin Huang ${ }^{1 \#}$, Youlin Tuo ${ }^{2}$, Yun Wang ${ }^{3}$ \\ ${ }^{1}$ Department of Outpatient, ${ }^{2}$ Department of Breast Surgery, ${ }^{3}$ Department of Neurology, Sichuan Academy of Medical Sciences \& Sichuan Provincial \\ People's Hospital, School of Medicine, University of Electronic Science \& Technology of China, Chengdu, China \\ Contributions: (I) Conception and design: J Xiang, S Huang; (II) Administrative support: Y Tuo; (III) Provision of study materials or patients: J Xiang, \\ S Huang, Y Tuo; (IV) Collection and assembly of data: All authors; (V) Data analysis and interpretation: J Xiang, S Huang, Y Wang; (VI) Manuscript \\ writing: All authors; (VII) Final approval of manuscript: All authors. \\ \#These authors contributed equally to this work and are co-first authors. \\ Correspondence to: Youlin Tuo. Department of Breast Surgery, Sichuan Academy of Medical Sciences \& Sichuan Provincial People's Hospital, School \\ of Medicine, University of Electronic Science \& Technology of China, No. 32, West Section 2, 1st Ring Road, Qingyang District, Chengdu, China. \\ Email: 99764156@qq.com; Yun Wang. Department of Neurology, Sichuan Academy of Medical Sciences \& Sichuan Provincial People’s Hospital, \\ School of Medicine, University of Electronic Science \& Technology of China, No. 32, West Section 2, 1st Ring Road, Qingyang District, Chengdu, \\ China. Email: lizzywon77@163.com.
}

Background As one of the main malignant tumors, breast cancer remains a worldwide public health issue. Here, we aimed to analyze the effects of breast-conserving surgery (BCS) combined with sentinel lymph node biopsy (SLNB) and axillary preservation on the recurrence, metastasis, complications, and cosmetic results of early breast cancer patients (BCPs).

Methods: The clinical data of 143 BCPs admitted to our hospital from January 2017 to January 2019 were collected retrospectively, and all patients were female. Patients (76 cases) undergoing BCS combined with SLNB and axillary preservation treatment were set as the combined group, and 67 cases undergoing traditional modified radical surgery were set as the control group. After the perioperative conditions of patients in the two groups were compared, the patients were followed up for 14 months to record information on the quality of life, recurrence, metastasis, complications, and cosmetic results.

Results: The operation time, intraoperative blood loss, and extubation time of the combined group were significantly less than the control group $(\mathrm{P}<0.05)$; the quality of life of patients in both groups improved after treatment, but the quality of life of patients in the combined group was significantly higher than that of the control group $(\mathrm{P}<0.05)$; both groups of patients were followed up for 14 months after treatment, and there were no deaths. The rates of local recurrence and distant metastasis were $2.98 \%$ and $5.97 \%$ in the control group and $5.26 \%$ and $6.57 \%$ in the combined group, respectively, showing no significant difference between the two groups $(\mathrm{P}>0.05)$; the incidence of postoperative complications was $14.92 \%$ in the control group, which was significantly higher than the incidence of $3.94 \%$ of the combined group $(\mathrm{P}<0.05)$; the proportion of postoperative cosmetic results with an excellent and good rating was $59.7 \%$ in the control group, which was significantly lower than the $93.42 \%$ reported in the combined group $(\mathrm{P}<0.05)$.

Conclusions: BCS combined with SLNB and axillary preservation provided good clinical and cosmetic results and can improve the quality of life of patients and reduce the rate of recurrence and metastasis.

Keywords: Breast-conserving surgery (BCS); sentinel lymph node biopsy and axillary preservation; early breast cancer; recurrence and metastasis; complications

(c) Gland Surgery. All rights reserved. 
Submitted Jun 04, 2020. Accepted for publication Jul 29, 2020.

doi: $10.21037 / g s-20-584$

View this article at: http://dx.doi.org/10.21037/gs-20-584

\section{Introduction}

Breast cancer, which stems from the epithelial tissue of the breast, is one of the most common malignant tumors that threaten women's life and health (1). Early diagnosis and early treatment are advocated in clinical practice, as they have a positive effect on the prognosis of patients. The treatment of breast cancer is mainly based on surgical treatment. In the past, conventional radical surgery was often used. However, the wide flap peeling range, large area of the breast trauma, high intraoperative bleeding, and extensive operation time are drawbacks of surgical treatment and can even result in death after surgery, especially for patients with low tolerance such as elderly patients or those with other medical diseases $(2,3)$. Even if the disease is cured after treatment, patients may still suffer emotionally from the lack of a breast after surgery, from lymph node edema, and from the limited motion of the affected limb, resulting in a poor prognostic quality of life. According to statistics, early breast-conserving surgery (BCS) has become the first choice of treatment for patients with early breast cancer in countries around the world. The treatment effect of BCS is no different from that of modified radical surgery, but can meet the aesthetic need of patients and improve prognosis $(4,5)$. In recent years, the treatment of BCS combined with sentinel lymph node biopsy (SLNB) has been widely used clinically because of its advantages in avoiding breast loss and keeping the patient's body intact to the greatest extent. However, whether this treatment has an impact on the prognosis of patients and recurrence is not clear yet. Therefore, this study retrospectively analyzed the clinical and follow-up data of 76 cases with early breast cancer patients treated with BCS combined with SLNB to protect the axillary. Compared with traditional modified radical mastectomy, we also summarized the effects of BCS combined with SLNB and axillary preservation on the recurrence, metastasis, complications, and cosmetic results of early BCPs.

We presented the following article in accordance with the STROBE reporting checklist (available at http://dx.doi. org/10.21037/gs-20-584).

\section{Methods}

\section{General information}

The clinical data of 143 BCPs admitted to our hospital from January 2017 to January 2019 were collected retrospectively. All patients were female. Among them, 76 patients who had undergone BCS combined with SLNB and axillary preservation treatment were set as the combined group, and were aged from 30 to 70 years old, with an average age of $51.32 \pm 8.55$ years old; meanwhile, 67 cases who had undergone traditional modified radical surgery were set as the control group, and were aged from 31 to 70 years old, with an average age of $51.69 \pm 8.79$ years old. There was no significant difference in the general data between the two groups $(\mathrm{P}>0.05)$, so they were considered comparable for other indicators. All patients were treated for the presence of unidentified masses, and were diagnosed and classified according to the tumor-node-metastasis (TNM) staging standard established by the International Union Against Cancer. Among the 143 patients, 107 cases were at stage I, and 36 cases were at stage II.

The inclusion criteria were the following: (I) all subjects were informed of the content of the study and agreed to participate; (II) all subjects met the breast cancer-related diagnostic criteria (6); (III) the maximum tumor diameter in subjects was $\leq 2 \mathrm{~cm}$, with the distance between the tumor and areola exceeding $2 \mathrm{~cm}$; (IV) preoperative clinical examination of subjects showed no lymphadenopathy at the armpit.

The exclusion criteria were the following: (I) patients with other diseases that could affect this study; (II) patients with infectious breast diseases; (III) patients with autoimmune diseases; (IV) patients undergoing relevant chemotherapy or radiotherapy treatment before surgery; (V) patients with other malignant tumors; (VI) patients who could not complete follow-up; (VII) patients with non-primary breast cancer. The study was approved by Sichuan Academy of Medical Sciences \& Sichuan Provincial People's Hospital (ChiECRCT20190184). All procedures performed in this study involving human participants were in accordance with the Declaration of Helsinki (as revised in 2013). The 
informed consent was taken from all the patients.

\section{Surgical methods}

In the combined group, patients underwent BCS combined with SLNB and axillary preservation treatment. After the patient was deeply anesthetized, the positions of sentinel lymph nodes above the tumor and under the armpit were selected for surgical incisions. The tumor and normal tissue of at least $1 \mathrm{~cm}$ around tumor visible to the naked eye were quickly frozen. For patients with breast invasive ductal carcinoma, the tissue was cut off at different directions below the internal and external basement of the primary tumor in the intraluminal cavity and sent rapidly for freezing. The diameter of the cut tissue needed to be 0.5 $\mathrm{cm}$ or greater. If there was residual tumor at the resection margin, the cut area was enlarged locally. When the new resection margin was negative, the BCS was performed. If it was still positive, the BCS was given up and a total mastectomy was performed. SLNB: The position of the sentinel lymph nodes was determined by the combination of preoperative indocyanine green tracer and intraoperative Meilan positioning. Before surgery, the indocyanine green tracer was used to locate and mark the position, accordingly. During the operation, $2-4 \mathrm{~mL}$ of methylene blue $(1 \%)$ was injected under the skin around the areola of the breast and around the tumor. After injection, the patient received a local massage for at least 5 minutes, and an incision was made at the location marked before surgery. The incision of the subsurface separation flap was conducted in the direction of axilla. The blue-stained lymphatic vessels could be found following the direction of the parallel muscle bundle outside the pectoralis major, and the chosen bluestained lymph nodes were the sentinel lymph nodes that required biopsy. After excision, the tissue was rapidly frozen. If it was detected to be negative, no axillary lymph node dissection was performed, while if it was positive, axillary lymph node dissection was performed. Postoperatively, the patient was compressively bandaged with a chest strap. All patients underwent adjuvant therapies, such as radiotherapy, chemotherapy, or endocrine therapy.

Patients in the control group underwent modified radical surgery. A fusiform incision was made, and the skin within $3 \mathrm{~cm}$ of the mass including the nipple was removed, and the patient's axillary lymph node tissue was removed but the pectoralis major and minor muscles was preserved. The axillary lymph node tissue was cleared in stage I patients, and patients in stage II were not allowed to retain the intercostal brachial nerve. After the operation, a chest strap was used to perform compression bandaging. Among the patients in the control group, 11 patients only underwent endocrine therapy, while the remaining patients received at least one of the adjuvant therapies of radiotherapy, chemotherapy, or endocrine therapy.

\section{Observation indicators}

After the perioperative conditions of patients in the two groups were compared, the patients were followed up for 14 months to record the quality of life, recurrence, metastasis, complications, and cosmetic effects of patients. The perioperative conditions included operation time, intraoperative blood loss, and extubation time. The cancer patient quality of life measurement scale was used to evaluate the quality of life before and after treatment and consisted of 5 items: physical, role, emotional, cognitive, and social function. The higher the score, the better the patient's quality of life. Recurrence and metastasis included local recurrence, distant metastasis, and death. Complications included subcutaneous effusion, mild skin flap necrosis, and upper limb edema on the affected side. The postoperative cosmetic effect was evaluated using the relevant standards of the United Radiation Therapy Center (7), and the results were divided into three levels: excellent, good, and poor.

\section{Statistical analysis}

The data of this study were analyzed by SPSS 23.0 software (IBM, USA). The measurement data were described by mean \pm standard deviation $\left(\bar{x}_{ \pm}\right)$, and analyzed using the $t$-test. Count data were tested by $\chi^{2}$ test. Results with a $\mathrm{P}$ value $<0.05$ were considered statistically significant.

\section{Results}

\section{Comparison of perioperative conditions between the two groups of patients}

The operation time, intraoperative blood loss, and extubation time of the combined group were significantly lower than those of the control group $(\mathrm{P}<0.05$, Table 1$)$.

\section{Comparison of the quality of life between the two groups of patients before and after treatment}

There was no significant difference in the quality of life 
Table 1 Comparison of perioperative conditions between the two groups $(\bar{x} \pm s)$

\begin{tabular}{|c|c|c|c|c|}
\hline Variable & Control group & Combined group & $\mathrm{t}$ & $\mathrm{P}$ \\
\hline Operating time (min) & $89.19 \pm 10.87$ & $65.33 \pm 10.36$ & 13.429 & 0 \\
\hline Intraoperative blood loss (mL) & $200.41 \pm 22.04$ & $89.33 \pm 9.82$ & 39.706 & 0 \\
\hline Extubation time $(\mathrm{d})$ & $25.69 \pm 6.23$ & $10.36 \pm 1.13$ & 21.071 & 0 \\
\hline
\end{tabular}

Table 2 Comparison of quality of life before and after treatment in the two groups $(\bar{x} \pm s)$

\begin{tabular}{|c|c|c|c|c|c|c|c|c|c|c|c|}
\hline Group & Cases & \multicolumn{2}{|c|}{ Physical function } & \multicolumn{2}{|c|}{ Role function } & \multicolumn{2}{|c|}{ Emotional function } & \multicolumn{2}{|c|}{ Cognitive function } & \multicolumn{2}{|c|}{ Social function } \\
\hline $\begin{array}{l}\text { Control } \\
\text { group }\end{array}$ & 67 & $60.23 \pm 8.11$ & $71.56 \pm 7.33^{\mathrm{a}}$ & $52.20 \pm 13.23$ & $60.20 \pm 11.04^{\mathrm{a}}$ & $64.36 \pm 9.76$ & $74.66 \pm 8.56^{a}$ & $76.66 \pm 8.56$ & $87.23 \pm 7.55^{a}$ & $66.26 \pm 9.84$ & $78.62 \pm 9.55^{a}$ \\
\hline $\begin{array}{l}\text { Combined } \\
\text { group }\end{array}$ & 76 & $60.69 \pm 8.26$ & $89.32 \pm 8.22^{a}$ & $52.01 \pm 13.39$ & $77.39 \pm 12.19^{a}$ & $64.58 \pm 9.68$ & $81.02 \pm 9.29^{a}$ & $76.02 \pm 8.19$ & $95.02 \pm 8.88^{a}$ & $66.22 \pm 9.12$ & $89.45 \pm 8.94^{a}$ \\
\hline$P$ & - & 0.738 & 0.000 & 0.932 & 0.000 & 0.892 & 0.000 & 0.648 & 0.000 & 0.979 & 0.000 \\
\hline
\end{tabular}

Note: "a" is the comparison between groups before and after treatment, $\mathrm{P}<0.05$.

Table 3 Comparison of postoperative recurrence and metastasis between the two groups (\%)

\begin{tabular}{lcccc}
\hline Variable & Control group & Combined group & $\chi^{2}$ & P \\
\hline Cases & 67 & 76 & - & 0.438 \\
Local recurrence & $2(2.98)$ & $4(5.26)$ & 0.022 & 0.508 \\
Distant metastasis & $4(5.97)$ & $5(6.57)$ & - & - \\
Death & 0 & 0 & & 0.881 \\
\hline
\end{tabular}

between the two groups before treatment $(\mathrm{P}>0.05)$. After treatment, the quality of life of patients in both groups were improved, and the improvement effect of the quality of life of the patients in the combined group was significantly better than that of the control group $(\mathrm{P}<0.05$, Table 2$)$.

\section{Comparison of postoperative recurrence and metastasis between the two groups}

No deaths occurred in either group of patients after 14 months of follow-up. The rates of local recurrence and distant metastasis were $2.98 \%$ and $5.97 \%$ in the control group and $5.26 \%$ and $6.57 \%$ in the combined group, respectively, and were not significantly different between these two groups $(\mathrm{P}>0.05$, Table 3$)$.

\section{Comparison of postoperative complications of patients} between the two groups

The incidence of postoperative complications in the control group was $14.92 \%$, which was significantly higher than that of the $3.94 \%$ in the combined group $(\mathrm{P}<0.05$, Table 4).

\section{Comparison of postoperative cosmetic effects between the two groups}

The proportions of excellent and good ratings for postoperative cosmetic result were $59.70 \%$ and $93.42 \%$ for the control group and the combined group, respectively $(\mathrm{P}<0.05$, Table 5). 
Table 4 Comparison of postoperative complications between the two groups (\%)

\begin{tabular}{lcccc}
\hline Variable & Control group & Combined group & $\chi^{2}$ & $P$ \\
\hline Cases & 67 & 76 & - & - \\
Subcutaneous hydrops & $2(2.98)$ & $1(1.31)$ & - & - \\
Mild skin flap necrosis & $3(4.47)$ & $0(0.00)$ & - & - \\
Edema of the affected upper limb & $5(7.46)$ & $2(2.63)$ & - & - \\
Sum & $10(14.92)$ & $3(3.94)$ & 5.193 & 0.023 \\
\hline
\end{tabular}

Table 5 Comparison of postoperative cosmetic effect between the two groups (\%)

\begin{tabular}{lcccc}
\hline Variable & Control group & Combined group & $\chi^{2}$ & $P$ \\
\hline Cases & 67 & 76 & - & - \\
Excellent & $20(29.85)$ & $42(55.26)$ & - & - \\
Good & $21(31.34)$ & $29(38.15)$ & - & - \\
Poor & $27(40.29)$ & $5(6.57)$ & - & - \\
Excellent and good rating & $40(59.70)$ & $71(93.42)$ & 23.309 & 0 \\
\hline
\end{tabular}

\section{Discussion}

Currently, the comprehensive mode of breast cancer treatment is mainly surgery, with endocrine therapy, chemoradiotherapy, and molecular targeted therapy as adjuvant therapy. The surgical treatment is the first and most important step, and the quality of the surgery is the determining factor as to whether the patient can continue with the follow-up treatment $(8,9)$. For the elderly or those with other medical diseases, the difficulty of surgery is greater, and the prognosis is not ideal. Therefore, choosing the right surgical method is the key to improving the prognosis of patients (10).

BCS has gradually become the gold standard of early stage breast cancer treatment, and its reliability has been supported by a significant amount of studies and clinical practice (11). However, due to the high recurrence rate of BCS, surgical treatment has been performed only tentatively in China. BCS is highly practical for single- and peripheraltype tumors, those with a diameter less than $2 \mathrm{~cm}$, and for patients with no lymph node metastasis in clinical examination and with the tumor stage I and II. It has been found that for the recurrence of breast cancer after BCS treatment, salvage mastectomy showed good effects, and the overall survival rate of patients increases following the salvage operations (12). It has been reported that the 5 -year survival rate of patients undergoing remedial radical surgery after BCS is not obviously different from those undergoing BSC again (13). It is also proposed that patients with breastconserving and axillary preservation surgeries who undergo mastectomy and tumor bed radiotherapy can effectively have reduced postoperative recurrence (14). SLNB is also a standard treatment for patients with early breast cancer. The safety and effectiveness of SLNB have been confirmed by clinical studies and related research. After treatment, the postoperative complications and recurrence rate of patients are low, and patients undergoing SLNB have a good prognosis of the lymph node situation of the axilla $(15,16)$.

Through prospective randomized trials, related studies have confirmed that BCS combined with SLNB in patients with lymph node-negative breast cancer has a better physical and psychological impact on patients than previous axillary lymph node dissection treatment, and lower occurrence rate of complications $(17,18)$. In this study, the results showed that patients undergoing BCS combined with SLBN and axillary preservation treatment experienced significantly lower operation times, intraoperative blood loss, and extubation times than did the control group, with the quality of life after treatment being better than that of the control group; also, $93.42 \%$ of patients in the combined group gave a good or excellent rating for the postoperative cosmetic result, which as significantly higher than that given by the control group $(\mathrm{P}<0.05)$; no significant 
difference was found in the recurrence and metastasis rate compared with the control group, which was consistent with the results of previous studies (19). Coupled with the findings of other related research, our study has confirmed that the combined treatment of BCS and SLNB improves the patient's perioperative condition, quality of life, and cosmetic result while maintaining good treatment effect and a low rate of recurrence and metastasis (20). There do exist absolute contraindications for BCS and axillary preservation including presence of multiple tumors, extensive cancerous gravel-like calcification, repeated positive enlarged resection margin, certain radiotherapy contraindications, and other conditions. Therefore, all the patients included in this study were confirmed by preexamination to have no axillary lymphadenopathy and none of the contraindications mentioned above.

In summary, early stage BCPs who undergo BCS combined with SLNB and axillary preservation treatment can enjoy good clinical and cosmetic effect, a reduced rate of recurrence and metastasis, and an improved the quality of life.

\section{Acknowledgments}

Funding: None.

\section{Footnote}

Reporting Checklist: The authors have completed the STROBE reporting checklist. Available at http://dx.doi. org/10.21037/gs-20-584

Data Sharing Statement: Available at http://dx.doi. org/10.21037/gs-20-584

Conflicts of Interest: All authors have completed the ICMJE uniform disclosure form (available at http://dx.doi. org/10.21037/gs-20-584). The authors have no conflicts of interest to declare.

Ethical Statement: The authors are accountable for all aspects of the work in ensuring that questions related to the accuracy or integrity of any part of the work are appropriately investigated and resolved. The study was approved by Sichuan Academy of Medical Sciences \& Sichuan Provincial People's Hospital (ChiECRCT20190184). All procedures performed in this study involving human participants were in accordance with the Declaration of Helsinki (as revised in 2013). The informed consent was taken from all the patients.

Open Access Statement: This is an Open Access article distributed in accordance with the Creative Commons Attribution-NonCommercial-NoDerivs 4.0 International License (CC BY-NC-ND 4.0), which permits the noncommercial replication and distribution of the article with the strict proviso that no changes or edits are made and the original work is properly cited (including links to both the formal publication through the relevant DOI and the license). See: https://creativecommons.org/licenses/by-nc-nd/4.0/.

\section{References}

1. Akram M, Iqbal M, Daniyal M, et al. Awareness and current knowledge of breast cancer. Biol Res 2017;50:33.

2. Dicks E, Roome R, Chafe J, et al. Factors influencing surgical treatment decisions for breast cancer: a qualitative exploration of surgeon and patient perspectives. Curr Oncol 2019;26:e216-25.

3. Whelan TJ, Julian JA, Berrang TS, et al. External beam accelerated partial breast irradiation versus whole breast irradiation after breast conserving surgery in women with ductal carcinoma in situ and node-negative breast cancer (RAPID): a randomised controlled trial. Lancet 2019;394:2165-72.

4. Winters ZE, Bernaudo L. Evaluating the current evidence to support therapeutic mammoplasty or breast-conserving surgery as an alternative to mastectomy in the treatment of multifocal and multicentric breast cancers. Gland Surg 2018;7:525-35.

5. De La Cruz L, Blankenship SA, Chatterjee A, et al. Outcomes After Oncoplastic Breast-Conserving Surgery in Breast Cancer Patients: A Systematic Literature Review. Ann Surg Oncol 2016;23:3247-58.

6. Spitz JA, Chao AH, Peterson DM, et al. Bioimpedance spectroscopy is not associated with a clinical diagnosis of breast cancer-related lymphedema. Lymphology 2019;52:134-42.

7. Wazer DE, Kaufman S, Cuttino L, et al. Accelerated partial breast irradiation: an analysis of variables associated with late toxicity and long-term cosmetic outcome after high-dose-rate interstitial brachytherapy. Int J Radiat Oncol Biol Phys 2006;64:489-95.

8. Naito Y, Urasaki T. Precision medicine in breast cancer. Chin Clin Oncol 2018;7:29.

9. Peart O. Breast intervention and breast cancer treatment 
options. Radiol Technol 2015;86:535M-558M; quiz 559-62.

10. Zhu J, Jiao D, Guo X, et al. Predictive factors and prognostic value of pathologic complete response of ipsilateral supraclavicular lymph nodes in breast cancer after neoadjuvant chemotherapy. Ann Transl Med 2019;7:666.

11. Weber WP, Soysal SD, Fulco I, et al. Standardization of oncoplastic breast conserving surgery. Eur J Surg Oncol 2017;43:1236-43.

12. Lee JH, Lee SK, Park SM, et al. Independent Prognostic Factors for Overall Survival after Salvage Operation for Ipsilateral Breast Tumor Recurrence Following BreastConserving Surgery. J Breast Cancer 2015;18:386-93.

13. van Maaren MC, de Munck L, Jobsen JJ, et al. Breastconserving therapy versus mastectomy in T1-2N2 stage breast cancer: a population-based study on 10-year overall, relative, and distant metastasis-free survival in 3071 patients. Breast Cancer Res Treat 2016;160:511-21.

14. Barbieri V, Sanpaolo P, Genovesi D. Interval between breast-conserving surgery and start of radiation therapy in early-stage breast cancer is not predictive of local recurrence: a single-institution experience. Clin Breast Cancer 2011;11:114-20.

15. Mi Y, Lv P, Wang F, et al. Efficacy, late complications, and cosmetic outcomes of targeted intraoperative radiotherapy

Cite this article as: Xiang J, Huang S, Tuo Y, Wang Y. Effect of breast-conserving surgery combined with sentinel lymph node biopsy and axillary preservation on the recurrence, metastasis, complications and cosmetic results of early breast cancer patients. Gland Surg 2020;9(4):1019-1025. doi: 10.21037/gs20-584 in breast-conserving surgery for early-stage breast cancer: a single-centre study in China. Jpn J Clin Oncol 2019;49:1120-5.

16. Charalampoudis P, Markopoulos C, Kovacs T. Controversies and recommendations regarding sentinel lymph node biopsy in primary breast cancer: A comprehensive review of current data. Eur J Surg Oncol 2018;44:5-14.

17. Ye F, Huang L, Lang G, et al. Outcomes and risk of subsequent breast events in breast-conserving surgery patients with BRCA1 and BRCA2 mutation. Cancer Med 2020;9:1903-10.

18. Gui Y, Liu X, Chen X, et al. A Network Meta-Analysis of surgical treatment in patients with early breast cancer. J Natl Cancer Inst 2019;111:903-15.

19. Folli S, Falco G, Mingozzi M, et al. Repeat sentinel lymph node biopsy in patients with ipsilateral recurrent breast cancer after breast-conserving therapy and negative sentinel lymph node biopsy: a prospective study. Minerva Chir 2016;71:73-9.

20. Gocer GPS, Ozer EE. Effect of radiotherapy on coronary arteries and heart in breast-conserving surgery: a dosimetric analysis. Radiol Oncol 2020;54:128-34.

(English Language Editor: J. Gray) 